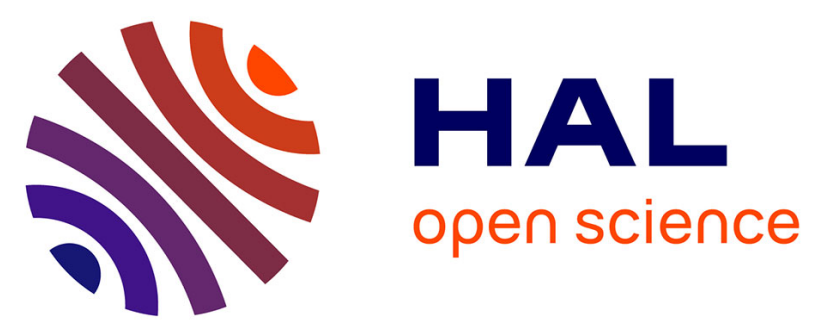

\title{
Sparse reconstruction from a limited projection number of the coronary artery tree in X-ray rotational imaging
} Ying Hu, Mi Youn Jung, Ahmed Oukili, Guanyu Yang, Jean-Claude Nunes, Jérôme Fehrenbach, Gabriel Peyré, Marc Bedossa, Limin Luo, Christine Toumoulin, et al.

\section{To cite this version:}

Ying Hu, Mi Youn Jung, Ahmed Oukili, Guanyu Yang, Jean-Claude Nunes, et al.. Sparse reconstruction from a limited projection number of the coronary artery tree in X-ray rotational imaging. IEEE International Symposium on Biomedical Imaging (ISBI'12), May 2012, Barcelone, Spain. pp.804-807. hal-00702613

\section{HAL Id: hal-00702613 \\ https://hal.science/hal-00702613}

Submitted on 8 Jan 2013

HAL is a multi-disciplinary open access archive for the deposit and dissemination of scientific research documents, whether they are published or not. The documents may come from teaching and research institutions in France or abroad, or from public or private research centers.
L'archive ouverte pluridisciplinaire HAL, est destinée au dépôt et à la diffusion de documents scientifiques de niveau recherche, publiés ou non, émanant des établissements d'enseignement et de recherche français ou étrangers, des laboratoires publics ou privés. 


\title{
Sparse reconstruction from a limited projection number of the coronary artery tree in $\mathrm{X}$-ray rotational imaging
}

\author{
Y. Hu, M. Jung, A. Oukili, G. Yang, J.-C. Nunes, J. Fehrenbach, G. Peyré \\ M. Bedossa, L. Luo, C. Toumoulin, L. D. Cohen, Fellow, IEEE.
}

\begin{abstract}
This paper deals with the 3D reconstruction of sparse data in X-ray rotational imaging. Due to the cardiac motion, the number of available projections for this reconstruction is equal to four, which leads to a strongly undersampled reconstruction problem. We address thus this illness problem through a regularized iterative method. The whole algorithm is divided into two steps. Firstly, a minimal path segmentation step extracts artery tree boundaries. Secondly, a MAP reconstruction comparing L0-norm and L1-norm priors is applied on this extracted coronary tree. The reconstruction optimization process relies on a separable paraboloidal (SPS) algorithm. Some preliminary results are provided on simulated rotational angiograms.

Index Terms - reconstruction, Maximum a posteriori (MAP), minimal path, non-local active contours, $\mathrm{X}$-ray rotational coronary angiography
\end{abstract}

\section{INTRODUCTION}

Rotational X-ray imaging devices provide a new way to explore the possibly existing artery pathologies enhanced after injection of a dye product, the complete range of projections (80 up to 150 ), giving a pseudo 3 -D view of the coronary tree with all the structures moving all together. However the challenge remains today the 3D coronary reconstruction that will provide the ground for a platform dedicated to the planning and execution of percutaneous coronary interventions. Due to the rotation of the source detector system, all the structures are moving and their features (including the most basic one, the intensity, e.g the attenuation) are varying over time and space. The object of interest, the coronary tree, is patient dependent (changes in its branching structure, complex-shaped vessels), has low nonstationary contrast and crossings, superimpositions in the image sequence planes represent additional difficulties to deal with. The coronary motions, determined by the myocardium contraction, can not be reduced to rigid transformations: strong deformations occur with slow and fast phases and movement inversions.

Y. Hu, G. Yang and L. Luo are with the Laboratory of Image Science and Technology, SoutEast University, Nanjing, China

A. Oukili, J.C. Nunes, and C. Toumoulin are with the French National Institute for Health and Medical Research (INSERM), U1099, Rennes F-35000, France, and with the Laboratoire Traitement du Signal et de l'Image (LTSI), Université de Rennes 1, Rennes F-35000, France. jean-claude.nunes@univ-rennesl.fr

C. Toumoulin, L. Luo, G. Yang and Y. Hu are also with the Centre de Recherche en Information Biomédicale Sino-Franais (LIA CRIBs), Rennes, F-35000, France.

L.D. Cohen, M. Jung and G. Peyré are with CEREMADE, UMR CNRS 7534, Université Paris Dauphine, F-75775 Paris Cedex 16, France.

J. Fehrenbach is with the Institut de Mathématiques (IMT) de Toulouse, F-31062 TOULOUSE CEDEX 09, France.

M. Bedossa is with CHU Rennes, Department of Cardiology, Rennes F-35000, France.
Different approaches have been proposed for this reconstruction depending on whether we consider the set of available projections or only the one acquired at the same phase of the cardiac cycle [1]. In the first option, the 3D+T motion is first estimated throughout the cardiac cycle to perform a motion compensated tomographic reconstruction at a given instant of the cardiac cycle using all the available projections [2]. The construction of this motion model generally relies on modeling techniques to extend the information to the time domain and is most often estimated from extracted 2D centerlines [3]. In the second option, the reconstruction is carried out from a limited number of views (typically 4 - 6 projections), corresponding to a single cardiac phase selected by ECG-gating. This reconstruction can be obtained by solving a static tomographic inverse problem. But the low number of projections leading to a severe angular undersampling, prior information is often introduced to improve the quality and accuracy of this reconstruction [4]. Other methods still make use of the epipolar geometry constraints and feature matching techniques to find corresponding structures in each view and recover their geometry [5].

We address here this illness problem considering background subtracted projections so that a vessel only 3D reconstruction can be performed, introducing thus a sparse representation of the data in the space domain. The reconstruction procedure goes through a preliminary segmentation stage and a $l_{p}$ norm regularization $(p<2)$ within a statistical MAP reconstruction algorithm. These two methods are described in Section II. Preliminary results are given in Section III on simulated data.

\section{METHOD}

The segmentation algorithm takes benefit of both minimal path and level set evolution methods to extract the coronary tree on each projection of the sequence. Then, the background is estimated to build background subtracted images and deal with sparse data. We address respectively, for comparison, the $l_{0}$ and $l_{1}$ norm regularizations within a maximum a posterior (MAP) estimation. The optimization process relies on a separable paraboloidal surrogate (SPS) algorithm which was introduced by Erdogan et al. for PET image reconstruction under Poisson statistics [6] and later for MSCT reconstruction [7]. SPS functions are applied here in the frame of a gaussian distribution data model. 


\section{A. Proposed segmentation method}

The minimal path technique introduced by Cohen and Kimmel [10] has been extensively used for the extraction of tree or tubular tree structures. This approach has several advantages such as finding global minima, fast computation, easy implementation, and more powerful incorporation of user input. On the other hand, level set evolution techniques have a nice advantage, which is that they capture and represent the boundary of object directly, not just some path running through their interior as in the minimal path approach. Recently, an active contour model [12] was proposed in the level set framework, which constrains the local homogeneity of image features. This is crucial to overcome the difficulty of segmentation due to image inhomogeneity that is often seen in medical images.

1) Minimal Paths: The minimal path technique characterizes a boundary extraction approach that globally minimizes a geodesic active contour energy between two user supplied end points. The model is formulated as

$\min _{\gamma \in \mathcal{A}_{x_{0}, x}}\left\{E(\gamma)=\int_{0}^{L} w+P(\gamma(s)) d s=\int_{0}^{L} \tilde{P}(\gamma(s)) d s\right\}$

where $\mathcal{A}_{x_{0}, x}$ is the set of all paths linking $x_{0}$ and $x, s$ represents the arc-length, and $w>0$ is a constant imposing regularity on the curve. $P>0$ is a potential cost function computed from the image, which takes lower values on the interesting features of the image.

To compute the solution associated with the source point $x_{0}$ of this problem, a Hamiltonian approach was proposed in [10]. This finds the geodesic weighted distance $U_{0}$ by solving the Eikonal equation: $\left\|\nabla U_{0}(x)\right\|=\tilde{P}(x), \forall x \in \Omega$, with $U_{0}\left(x_{0}\right)=0$. Then, the minimal path $\gamma$ can be retrieved with a simple gradient descent on $U_{0}$ from $x$ to $x_{0}$, by solving the ordinary differential equation: $\frac{d \gamma(s)}{d s}=-\nabla U_{0}(\gamma(s))$ with $\gamma(0)=x$. To solve the Eikonal equation, one can use the Fast Marching algorithm [10] due to its lower complexity.

2) Non-local Active Contours: The non-local active contours model [12] aims at finding a contour that represents the boundary of object of interest, and minimizes the energy incorporated with a level set function $\phi: \Omega \rightarrow \mathbb{R}$,

$F(\phi)=\int_{\Omega} \int_{\Omega} \rho(H(\phi(x)), H(\phi(y))) K(x, y) d x d y+\gamma L(\phi)$ where $K(x, y)=G_{\sigma}(x-y) d\left(p_{x}, p_{y}\right)$ with $G_{a}(t)=$ $e^{-\|t\|^{2} / 2 a^{2}}$, a patch $p_{x}$ around the pixel $x$ and a metric $d(\cdot, \cdot) \geq 0$ that accounts for the similarity between patches, and where $L(\phi)=\int_{\Omega}\|\nabla H(\phi(x))\| d x$. $H$ is a smoothed Heaviside function, and $\rho$ is an indicator function such that $\rho(u, v)=1$ if $u=v, 0$ otherwise (e.g. $\rho(u, v)=$ $u v+(1-u)(1-v))$. The parameter $\sigma>0$ controls the scale of the local homogeneity one requires for the segmented object. The second term enforces the smoothness of the contour, thus $\gamma>0$ is a regularization parameter.

The solution of the minimization problem is computed by solving the evolution equation for an artificial time $t \geq 0$ : $\frac{\partial \phi(t, x)}{\partial t}=-\nabla F(\phi(t, x))$ with $\phi(0, x)=\phi_{0}(x)$. See [12] for more details.
3) Proposed Segmentation Algorithm: In the proposed algorithm, the minimal path technique is first used for an initial estimation of the tubular tree structure. The non-local active contours model is then performed, to make a level set function constructed from the minimal paths move towards the boundary. Thus, the segmentation algorithm is as follows: - (Estimation step) Given $x_{0}$ and end points $\left\{x_{i}\right\}_{i=1}^{k}$,

- Compute minimal paths $\gamma_{x_{0}, x_{i}}(i=1, \ldots, k)$.

- Compute an initial level set function $\phi_{0}$ as a signed distance function along the minimal paths.

- (Correction step) Solve the non-local segmentation model starting with the initial level set function $\phi(0, \cdot)=\phi_{0}$.

\section{B. $3 D$ reconstruction stage}

Rotational imaging provides a sequence of $2 \mathrm{D}$ projections acquired under continuous rotation of the $\mathrm{C}$-arm. The acquisition trajectory is limited to a circular arc with a fixed caudo-cranial angulation. Let consider $Y=\left\{Y_{1}, Y_{2}, \ldots, Y_{L}\right\}$ to be the projection images acquired at primary angles $\theta=\left\{\theta_{1}, \theta_{2}, \ldots, \theta_{L}\right\}, L$ being the number of considered rotation angles. If we assume the cardiac motion is relatively regular and periodical, then through ECG gating, we can choose a set of projections $Y^{s}$, which corresponds to the same 3D heart motion (i.e the same cardiac phase $s$ ). The 3D coronary tree $f^{s}$ at a given instant $s$ of the cardiac cycle, will be reconstructed from a very few number of projections, which renders the problem strongly ill-posed. We will further simplify the notation by using $Y$ instead of $Y^{s}$ for conciseness. Projections $Y$ are non-subtracted i.e they include both the background tissues and the coronary tree enhanced with the contrast agent. Thus, the expectation of the measurement $E(Y)$ can be written under the following form:

$$
\bar{Y}_{i}=\bar{Y}_{b_{i}} \exp \left(-[A . f]_{i}\right)
$$

with $[A f]_{i}=\sum_{j} a_{i j} f_{j}(j=1, \ldots, J, i=1, \ldots, I)$ and $(J, I)$ being the voxel and pixel numbers in the volume and on the detector plane respectively. $A$ is the cone beam projection operator, $a_{i j}$ denotes the contribution of voxel $j$ in the computation of pixel $i$, and $Y_{b_{i}}$ is the $i_{t h}$ element of the background tissues $Y_{b}$. The logarithm application on eq. 1 provides a new image that only contains the structure of interest: $\bar{g}_{i}=[A . f]_{i}$ with $\bar{g}_{i}=-\log \frac{\bar{Y}_{i}}{\bar{Y}_{b_{i}}}$. The data are thus supposed to follow a Gaussian distribution model:

$$
P\left(g_{i} \mid f\right)=\frac{1}{\sigma_{i} \sqrt{2 \pi}} \exp \left(-\frac{1}{2}\left(\frac{g_{i}-\overline{g_{i}}}{\sigma_{i}}\right)^{2}\right)
$$

1) MAP model: According to the Bayesian theory, the estimate of the unknown object $f$ is computed as a function which maximizes the posterior density $P(f \mid g)$ and is given by:

$$
\widehat{f}(g)=\arg \min _{f \geq 0}(-\log P(g \mid f)-\log P(f \mid \beta))
$$

The log-likelihood function $\log P(g \mid f)=L(g \mid f)$ is:

$$
L(g \mid f)=-\frac{1}{2}(g-\hat{g})^{T} \Sigma^{-1}(g-\hat{g})^{T}-\mathrm{const}
$$


where $\hat{g}=A f$ and $\Sigma$ is the covariance matrix: $\Sigma=\operatorname{diag}\left\{\sigma_{i}\right\}^{2}$ with $\sigma_{i}^{2}=\max \left(\sigma_{\text {min }}^{2}, \log (\hat{g})\right)$. The prior function has the form of a Gibbs distribution $P(f \mid \beta)=\frac{\exp (-\beta R(f))}{Z(\beta)}, Z(\beta)$ being a scaling constant. Thus, $\log P(f \mid \beta)=-\beta R(f)$ with:

$$
R(f)=\sum_{j=1}^{J} \sum_{k \in N_{j}} \omega_{j k} \psi\left(f_{j}-f_{k}\right)
$$

where $N_{j}$ defines the neighborhood of the $\mathrm{j}^{\text {th }}$ voxel, and $\omega_{j k}$ is a positive value that expresses the interaction degree in clique $k j . \psi(t)$ is a symmetric function that penalizes the pairwise differences between neighbouring voxels. Various kinds of potential function $\psi$ have been proposed in the literature that are $l_{2}$ norm [4], $l_{1}$ norm [3] and $l_{0}$ norm [4]. We compare 2 of these priors: $l_{0}$ norm and $l_{1}$ norm respectively defined by: $\psi(t)=|\operatorname{sgn}(t)|$ and $\psi(t)=|t|$. The $l_{0}$ norm is non convex and not continuous around zero. This brings some difficulty for the optimization. A way for solving it is to go through surrogate Functions. Any function satisfying: $\lim _{\rho \rightarrow 0} \psi(t, \rho)=|\operatorname{sgn}(t)|$ can be taken as surrogates, such as $\psi(t, \rho)=\frac{2}{\pi} \arctan \frac{|t|}{\rho}$.

2) Optimization: The key of the SPS method is to find a decomposition of the objective function into a simple quadratic form that simplifies its optimization, guaranties a faster global convergence and reduces the computational time [5]. We define: $L(\hat{g})=\sum_{i} L_{i}\left(\hat{g}_{i}\right)=\sum_{i} \frac{\left(g_{i}-\hat{g}_{i}\right)^{2}}{2 \sigma_{i}{ }^{2}}$. Then $\tilde{L}_{j}\left(f_{j}, \hat{f}\right)=\sum_{i} L_{i}\left(g_{i}, \hat{g}_{i}\right)$. The voxel based quadratic likelihood function is then given by $\tilde{Q}_{j}\left(f_{j}\right)=\tilde{L}_{j}(\hat{f})+$ $\dot{L}_{j}\left(f_{j}\right)\left(f_{j}-\hat{f}_{j}\right)-\frac{1}{2} d_{j}\left(f_{j}-\hat{f}_{j}\right)^{2}$ with $\dot{L}_{j}\left(f_{j}\right)=\sum_{i} a_{i j} \dot{L}_{i}\left(\hat{g}_{i}\right)$ and $d_{j}=\sum_{i} a_{i j}{ }^{2} \sigma_{i}{ }^{2}$. In the case of the non convex prior, we apply an update into 2 steps: $f_{j}{ }^{k+1,1}=\max \left(0, f_{j}{ }^{k}-\frac{\dot{Q}_{j}^{k}}{\ddot{Q}_{j}^{k}}\right)$ and $f_{j}{ }^{k+1,2}=\max \left(0, f_{j}{ }^{k+1,1}+\beta \frac{\partial R(f)}{\partial f_{j}}\right)$.

\section{RESULTS}

Evaluations have been carried out on simulated data obtained from a 3D dynamic sequence acquired on a 64-slice GE LightSpeed CT scan [9]. A sequence of 20 3D binary coronary trees was built corresponding to 20 different cardiac phases. A C-arm rotational R-X coronary angiography was then simulated using the Siemens Axiom System imaging protocol. The detector plane $(200 \mathrm{~mm})^{2}$ was uniformly sampled into 512 pixels. Reconstructions were performed within a volume of $(110 \mathrm{~mm})^{3}, 80$ projections of the 3D binary tree were generated, uniformly spaced over the range RAO $90^{\circ}$ to LAO $90^{\circ} .4$ cardiac cycles were considered, this means that a volume (associated with a phase $s$ of the cardiac cycle) was projected 4 times during the acquisition, according to different viewpoints. The projection operator $A$ has been computed according to [8]. We used then the method of low order polynomials approximation to build background tissues images from the CT scan datasets. Fig. 1 displays four simulated projections $Y^{s}, s$ being the phase 0 and the projection angles RA0 $90^{\circ}$, RAO $30^{\circ}$, LAO $30^{\circ}$ and LAO $90^{\circ}$.
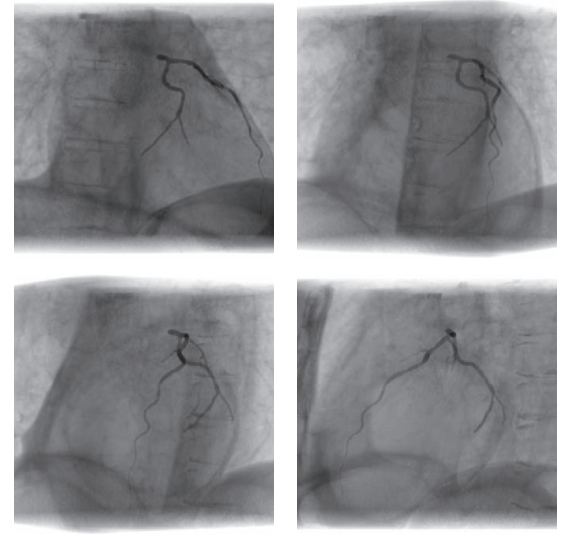

Fig. 1. Simulated projections at phase 0 . Projection angles are respectively (RA0 $90^{\circ}$, RAO $30^{\circ}$, LAO $30^{\circ}$ and LAO $90^{\circ}$ ).
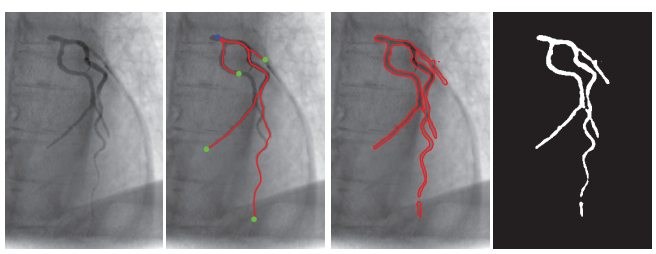

Fig. 2. Segmentation results. (1st) original image, (2nd-4th) minimal paths, resulting curves of NLAC, extracted vessels. Blue and green points represent a starting point and end points respectively.

\section{A. Segmentation results}

The fig. 2 presents segmentation results of the algorithm described in II-A. For the potential $P$, we computed an enhanced image $f^{0}=G_{b} * f-f$ with $b=10$, which cleaned the original image $f$ by subtracting the smoothly varying background. We used $\tilde{P}(x)=w+\left|f^{0}(x)-\max _{x} f^{0}(x)\right|$ with $w=0.001$. For the non-local active contours, we used the $L^{2}$ distance $d$ based on the image $f$ with patches of size $3 \times 3$ pixels, $G_{\sigma}$ of size $15 \times 15$ pixels with $\sigma=1000$, and we set $\gamma=40$. The curve evolution method could correct erroneously segmented (or missing) parts that are initially estimated by the minimal path technique.

\section{B. Reconstruction results}

Background images $Y_{b}$ were then estimated by subtracting the segmented images (fig. 3) from original projections $Y$ and applying an inpainting method on $Y_{b}$ to interpolate the missing background data (at the coronary tree location). The background segmented images $g$ are then obtained by the logarithmic subtraction $\left(\log Y_{b}-\log Y\right)$.

The hyperparameter $\beta=0.002$ for $l_{0}$ norm, $\beta=0.0005$ and for $l_{1}$ norm. We applied a relaxation scheme to estimate the optimal value $\rho$ in the $l_{0}$ norm prior computation. Fig. 4 displays the 3D coronary reconstruction obtained from background segmented images. The reconstruction quality is evaluated using DICE overlap criterion. This criterion is equal to 0.42 for both $l_{0}$ and $l_{1}$ norms. For comparison, we performed the reconstruction of ideal coronary trees directly 

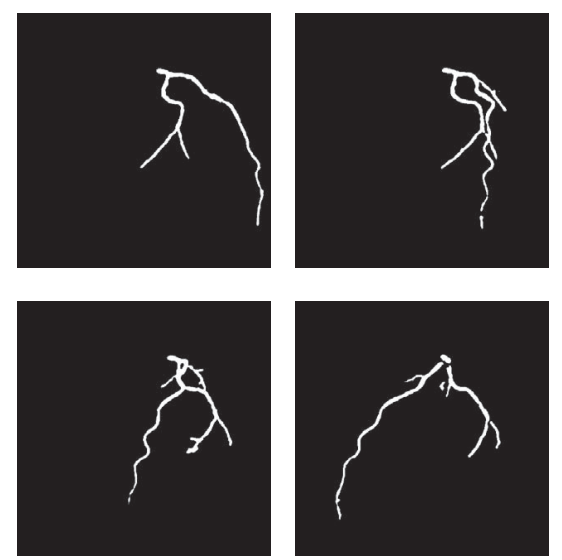

Fig. 3. Segmented images $g$ for the four projections of the phase 0 of the cardiac cycle.

obtained from the 3D reference sequence and got a DICE of 0.99 for $l_{0}$ norm and 0.97 for $l_{1}$ norm. This result shows that the reconstruction algorithm is highly dependent on the segmentation-based background estimation.

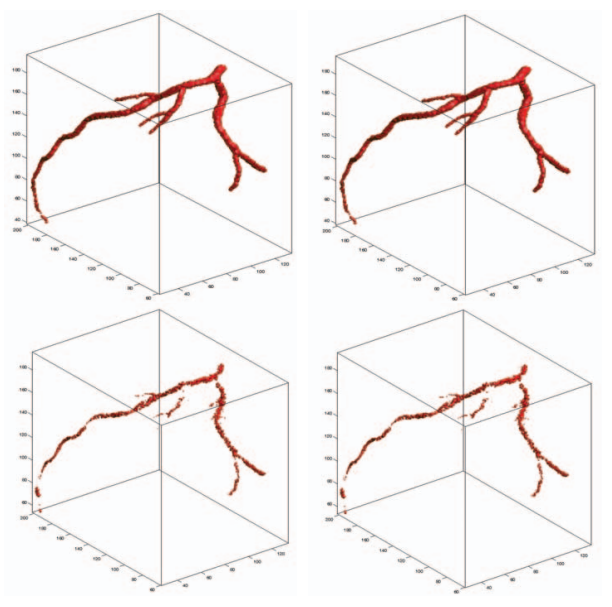

Fig. 4. Coronary tree reconstruction from the 4 projections depicted in the fig. 1. First line: reconstruction from the ideal tree (left: with $l_{0}$ norm, right: with $l_{1}$ norm). Second line: reconstruction from the background segmented images (left: with $l_{0}$ norm, right: with $l_{1}$ norm).

\section{CONCLUSION}

The coronary artery tree reconstruction from a very few number of projections appears to be a very strong ill-posed problem. Indeed the cardiac motion leads to a severe angular undersampling. Moreover, we restricted the projection number to four for the static reconstruction in order to position ourself in situation of clinical routine where the acquisition is made over a period of 4 seconds. We proposed to solve this problem by simplifying the image contents with a preliminary segmentation stage, which is based on minimal paths and non local active contours to build background subtracted projections. We compared then $l_{0}$ and $l_{1}$ norm regularizations within a statistical MAP reconstruction algorithm to deal with the spatial sparsity of the data. Evaluations have been performed by means of a realistic phantom representing an arterial tree extracted from a sequence of MDCT datasets in order to get a ground truth. Preliminary results make appear that the reconstruction algorithm is very sensitive to the quality of the segmentation and the background estimation. Our further objective will thus be to improve the background estimation as well as to work on a new prior that will better discriminate the coronary/background structures during the reconstruction process.

\section{ACKNOWLEDGMENTS}

This work was supported by ANR grant MESANGE ANR-08-BLAN-0198 and the National Natural Science Foundation NO. 31100713.

\section{REFERENCES}

[1] G. Schoonenberg, A. Neubauer, and M. Grass, "Three-Dimensional coronary visualization, Part 2: 3D reconstruction", Cardiology Clinics, Advances in coronary Angiography, vol. 27, no. 3, pp. 453-465, 2009.

[2] D. Schäfer, J. Borgert, V. Rasche, and M. Grass, "Motion compensated and gated cone beam filtered back projection for 3D rotational X-ray angiography", IEEE Trans. Med. Img., vol. 25, no. 7, pp. 898-906, 2006.

[3] A. Bousse, J. Zhou, G. Yang, J-J. Bellanger, and C. Toumoulin, "Motion compensated tomography reconstruction of coronary arteries in rotational angiography", in IEEE Trans. Biomed. Eng., vol 56(4), 2009, pp 1254-1257

[4] E. Hansis, D. Schäfer, O. Dössel, and M. Grass, "Evaluation of iterative sparse object reconstruction from few projections for rotational coronary angiography", in IEEE Trans.Med. Img., vol. 27(11), 2008, pp 1548-1555.

[5] S. Zheng, T. Meiying, and S. Jian, "Sequential reconstruction of vessel skeletons from X-ray coronary angiographic sequences", Computarized Medical Imaging and Graphics, 34(5), 2010, pp 333-345

[6] H. Erdogan, and J. Fessler, "Ordered subsets algorithms for transmission tomography", Physics in Medicine and Biology, vol. 44, pp. 2835-2851.

[7] Y. Hu, L. Xie, L. Luo, J.-C. Nunes, and C. Toumoulin, "L0 constrained sparse reconstruction for multi-slice helical CT reconstruction", Physics in Medicine and Biology, 56, vol. 4, pp. 1173-89, 2011.

[8] B.D. Man and S. Basu, "Distance-driven projection and backprojection in three dimensions", Phys. Med. Biol., vol. 49, pp 2463-2475, 2004.

[9] G. Yang, A. Bousse, C. Toumoulin, and H. Shu, "A multiscale tracking algorithm for the coronary extraction in MSCT angiography", Proc. Eng. Med. Biol. Soc. (EMBS), vol. 1, pp 3066-3069, 2006.

[10] L.D. Cohen and R. Kimmel, "Global minimum for active contour models: A minimal path approach", International Journal of Computer Vision, vol. 24, no. 1, pp. 57-78, 1997.

[11] J. Sethian, "Fast marching methods", SIAM Review, vol. 41, no. 2, pp. 199-235, 1999.

[12] M. Jung, G. Peyré, and L.D. Cohen, "Nonlocal active contours", Proc. SSVM'11, Ein Gedi, Israel, June 2011. 